\title{
OC-061 \\ EMERGENCY ADMISSION AS A ROUTE FOR OESOPHAGOGASTRIC CANCER DIAGNOSIS: A MARKER OF POOR OUTCOME AND A CANDIDATE QUALITY INDICATOR FOR LOCAL SERVICES
}

doi:10.1136/gut.2011.239301.61

M Shawihdi, ${ }^{1, *}$ N Stern, ${ }^{2}$ E Thompson, ${ }^{3}$ R Sturgess, ${ }^{2}$ N Kapoor, ${ }^{2}$ M G Pearson, ${ }^{3}$ K Bodger ${ }^{1}{ }^{1}$ Department of Gastroenterology and Nutrition, University of Liverpool, UK; ${ }^{2}$ Digestive Diseases Unit, University Hospital Aintree, UK; ${ }^{3}$ Aintree Health Outcomes Partnership, University of Liverpool, Liverpool, UK

Introduction The UK National Cancer Plan (2000) introduced a 'two week' waiting time standard for suspected malignancy and guidance to encourage early diagnosis. Improved access to elective (ELECT) investigation should reduce the need for emergency (EMERG) admission. This study examined route of diagnosis and outcomes for oesophagogastric cancer (OGC), both locally and nationally.

Methods Local OGC cases were audited for 2-year periods before ('Pre': July 97-June 99) and after ('Post': Jan 01-Dec 02) service re-design, collecting details of demographics, tumour type, stage, dates of referral, diagnosis, treatment and survival. Within a project funded by the NHS Information Centre, we developed novel linkage algorithms to analyse Hospital Episode Statistics for England (2006-2008) and methods to track OGC care chronologically, selecting only incident cases with a valid pathway of coded diagnostic and therapeutic interventions. External linkage to death registry established date of death and 2-year survival.

Results LOCAL DATA: $\mathrm{n}=333$ cases (Pre, $\mathrm{n}=152$; Post, $\mathrm{n}=181$ ). No change in \% of patients diagnosed via EMERG route after service re-design (Pre: $30.9 \%$ vs Post: $31.5 \%$; 
$\mathrm{p}=0.981)$, nor any change in age, symptom or tumour profile of EMERG cases. Local EMERG cases were older than ELEC (75 vs 68 years; $p<0.0001)$, less likely to have potentially curative treatment $(13.5 \%$ vs $40 \%$; $<<0.0001)$ and had poorer 3 -year survival (10.6\% vs $22.2 \%$, p = 0.013). EMERG cases with dysphagia and/or weight loss had lower 3 year survival than those with other presenting features $(p=0.035)$.

NATIONAL DATA We identified 33,115 patients with OGC, of whom 26,097 (79\%) met study criteria. Of these, $7082(27 \%)$ were EMERG and 19,015 ELEC (73\%). EMERG cases were older (74 years vs 70 years; $p<0.001)$, less likely to undergo surgery (516 [2\%] vs 3780 [14.5\%], p < 0.001) and had poorer 2 -year survival (19.6\% vs $32.9 \%$, p < 0.001). The $\%$ of EMERG cases varied widely between cancer networks (22\% to $40 \%$ ).

Conclusion Findings are consistent with a recent report by the National Cancer Intelligence Network (Nov 2010) suggesting that a quarter of major cancers are diagnosed via the EMERG route. Our national linkage study suggests $27 \%$ of new OGC cases in England are diagnosed as EMERG and this mode of presentation predicts a poor outcome, confirmed by detailed local audit. Although EMERG admission is unavoidable for some cases, the observed variation across the country suggests possible unresolved inequalities in patient access. Monitoring of this candidate indicator could assess the impact of new initiatives to promote earlier elective diagnosis.

Competing interests None.

Keywords admission rates, diagnosis, gastric cancer, guidelines, mortality, oesophageal cancer, service provision. 